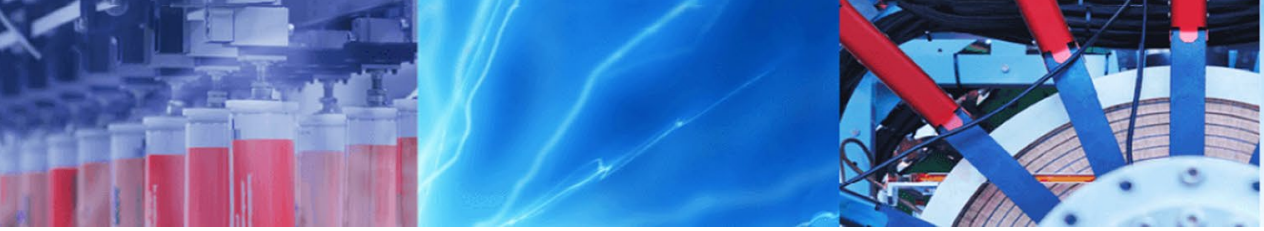

Research Article

\title{
Non-invasive blood glucose concentration level estimation accuracy using ultra-wide band and artificial intelligence
}

\author{
Minarul Islam ${ }^{1} \cdot$ Md Shawkat Ali $^{2} \cdot$ Nusrat Jahan Shoumy $^{3} \cdot$ Sabira Khatun $^{1}$ (D) $\cdot$ Mohamad Shaiful Abdul Karim ${ }^{1}$. \\ Bifta Sama Bari ${ }^{1}$
}

Received: 9 October 2019 / Accepted: 10 December 2019 / Published online: 28 January 2020

(c) Springer Nature Switzerland AG 2020

\begin{abstract}
Diabetes becomes a rapidly increasing global epidemic and getting serious health concern worldwide. There is no remedy except systematic management to keep blood glucose level under control. To achieve that regular glucose level monitoring is a routine task for a patient. This involves collection of blood physically from body with some discomfort and measuring using some device. To overcome this disadvantages and distress, non-invasive blood glucose measurement system is in demand. This article presents an ultra-wide band (UWB) microwave imaging and artificial intelligence based prospective solution to detect blood glucose concentration level non-invasively (without physical blood). The system consists of a pair of small UWB biomedical planar antenna, UWB transceiver as hardware and an artificial neural network with signal acquisition and processing interface as software module. The UWB signal with center frequency of 4.7 GHz was transmitted through ear lobe and forward scattering signals were received from other side. Characteristics features of received signal were extracted for pattern recognition and detection through deep artificial neural network. The system exhibits around $88 \%$ accuracy to detect glucose concentration in blood plasma. Besides, it is affordable, safe, user friendly and can be used with comfort in near future.
\end{abstract}

Keywords UWB technology · Non-invasive blood glucose measurement · Deep artificial neural network

\section{Introduction}

Diabetes is a chronic condition that occurs when the body unable to produce enough insulin or failed to use the produced insulin effectively. Insulin is a hormone which regulates blood glucose level by delivering glucose to the body cell to live. If the glucose cannot enter into the cells to be burnt as energy, it remains in the blood to cause diabetes. Over time, high blood glucose can affect major organs of the body which may led to serious health incidence including strokes, heart attacks, kidney failure, nerve damage, blindness, infections etc. There are two main types of diabetes. In case of Type-1 diabetes, insulin is not produced by the body at all and therefore need to inject insulin regularly for whole life span of a person. People with Type- 2 diabetes, usually body can produce required insulin until certain age but reduce overtime or unable to utilize properly as they grow older [1]. Thus two medical terms hypoglycaemia (reduced glucose level) and hyperglycaemia (high glucose level) can cause severe damage to almost whole body system, which often leads to serious body consequences.

Diabetes and its complications are the major causes of death in most countries [2]. According to International diabetes federation, it caused 5 million death with health care cost of $\$ 1197$ billion worldwide in 2015 and by 2040 there

$\triangle$ Sabira Khatun, sabirakhatun@ump.edu.my | ${ }^{1}$ Faculty of Electrical \& Electronics Engineering Technology, Universiti Malaysia Pahang, Pekan, Pahang, Malaysia. ${ }^{2}$ Universiti Malaysia Perlis, Kangar, Perlis, Malaysia. ${ }^{3}$ School of Computing and Mathematics, Charles Sturt University, Wagga Wagga, NSW, Australia. 
will be around 642 million diabetes patients worldwide [2]. Despite of the increasing rate, there is lack of immunization and treatment to prevent this disease thus frequent measurement and monitoring of blood glucose become an essential part of diabetic management. Present method of measuring is invasive using commercial glucometer. A blood sample is needed to be drawn either from vein or by pricking the fingertip and analysis by glucometer device [3]. This process is painful and creates discomfort for the patients specially who need to perform it several times a day to have a good metabolic body control. Thus development of a reliable non-invasive continuous glucose monitoring technique is in demand for last four decades. A good number of non-invasive technologies were invented but failed to retain required standard of accuracy thus couldn't survive in the market due to inconsistent result. Vashist [4] and Chi-Fuk So et al. [5] comprehensively analyzed most of those technologies along with advantages, disadvantages and future challenges. It was obligatory for the researcher to pursuit and attempted in for new technologies. Some recent non-invasive technologies includes Nuclear magnetic resonance spectroscopy [6], Electrocardiogram signal [7], Graphene based nanosensor [8], Saliva analysis [9], Thermal spectrum measurement [10], Breath acetone analysis [11], Impedance spectroscopy [12], Scattering spectroscopy [13] etc. which could attain initial success. GlucoTrack DF-F from Integrity applications Inc [14] claims to achieve high accuracy of measuring blood glucose concentration level (BGCL) non-invasively with combination of three technologies (ultrasonic, electromagnetic and thermal). The system is quite expensive and need periodical calibration.

In the field of exploring potential non-invasive and safe solutions another alternative can be ultra-wide band (UWB) imaging technique with artificial intelligence (AI) which have already been used successfully to diagnose various diseases including early cancer and/or BGCL [15-24]. This type of system relies on changes in dielectric properties (permittivity and conductivity) of target tissues or cells. UWB technology has significant advantage due to its easy penetration and non-ionizing nature [16]. In [23, 24], authors presented a proof of concept by using UWB with traditional artificial neural network (ANN) to detect $B G C L$ through hand tissues. Inspired by $[23,24]$, the performance of UWB along with deep artificial neural network (DANN) is investigated here to observe the change of blood dielectric properties as a function of both blood glucose concentration and frequency. Ear lobe is used here as a body part for better performance. The rest of the paper is organized as follows. The proposed method with system development is presented in Sect. 2, followed by the results and discussion in Sect. 3 , and conclusion at the end of this article.

\section{The proposed system}

\subsection{System work flow}

Usually, the received wave forms of an UWB signal pulse through different objects are changed due to variation of objects dielectric properties (permittivity, conductivity). The value of permittivity and conductivity decrease when the glucose concentration increases and the difference is more apparent in higher frequency range [17]. Thus the changes of dielectric properties of blood plasma can be considered as a function of both glucose concentration and frequency [18]. By keeping this in mind, we have conducted experimental investigation on a wide range of people as follows.

A pair of homemade UWB Micro strip patch bio-antenna was used for this experiment. Here, bi-static radar technique was followed using a transmitting antenna ( $\mathrm{Tx}$ ) and receiving antenna ( $\mathrm{Rx})$ based system model as shown in Fig. 1. Tx antenna transmitted UWB pulse train through human ear lobe, forward scattered signals were received by $\mathrm{Rx}$ antenna. Experimental system setup is shown in Fig. 2.

Received UWB waveforms have different values than the ideal waveform, which were considered for pattern recognition purpose. The total number of received wave forms were 208 collected from the human subjects for this experiment.

Human earlobe contains small amount of fat with no bone having many nerve endings which are bundled along with a number of capillary blood vessels. It does not contain cartilage thus has sufficient blood supply with relatively relaxed flow. Hence, the earlobe is a very convenient place on the body to measure blood glucose levels [19].

\subsection{The integrated system}

A Pair of UWB antenna was connected with UWB transceivers through coaxial cables as hardware part of the system. To receive and analysis signal data, a computer was connected to transceiver through a soft interface and Ethernet cable. The software part of the system resides here including signal processing, feature extraction and recognition. The integrated system is shown in Fig. 3.

\subsection{Hardware}

Main hardware used to integrate the whole system was UWB antenna and transceiver. To obtain better performance, a pair of custom made UWB rectangular patch 
Fig. 1 Theoretical system model

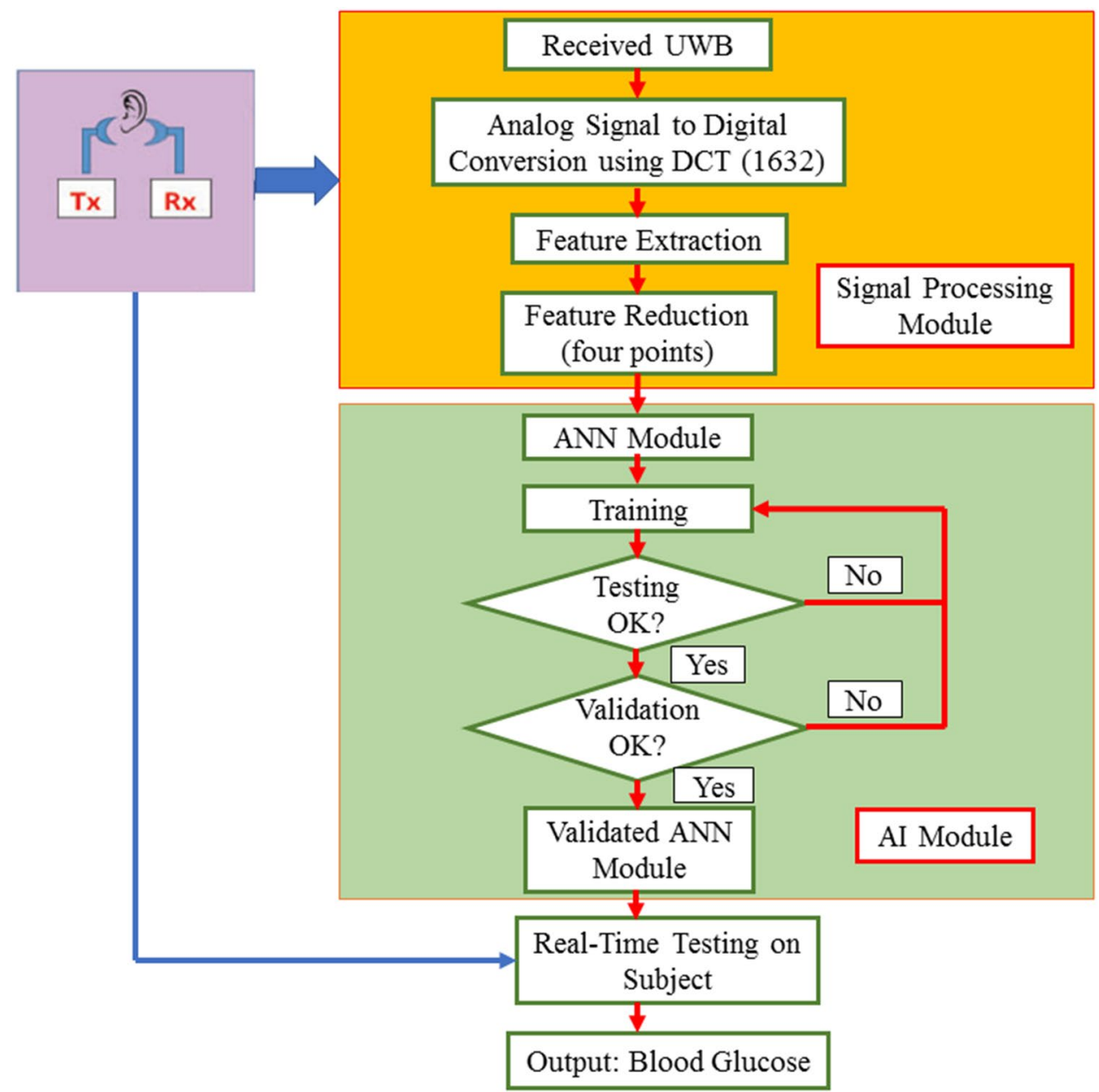

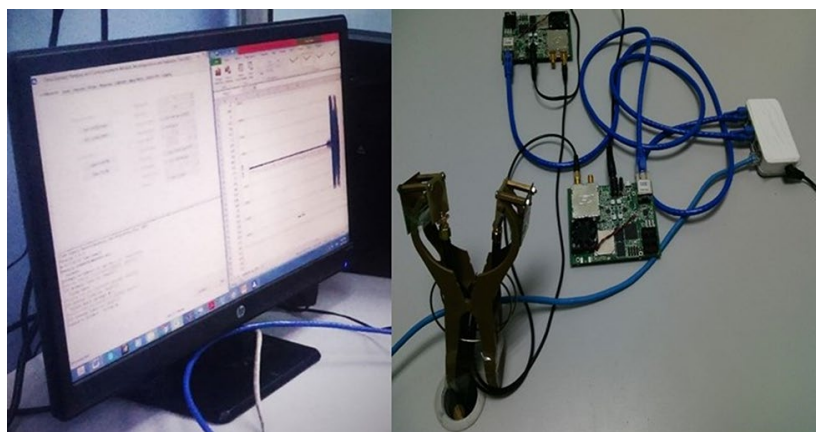

Fig. 3 Integrated system

the antenna in three levels to maintain the gain and directivity as per need [20]. The antenna was optimized and simulated using Agilent advanced design system (ADS) and computer simulation technology (CST) software. Then fabricated on FR4 and tested using Agilent PNA (the design details can be seen in [20]). The measured bandwidth, gain and directivity of the antenna are $8.77 \mathrm{GHz}$ ( $3.23 \mathrm{GHz}$ to $12 \mathrm{GHz}$ ), $6.09 \mathrm{~dB}$ and $8.15 \mathrm{dBi}$ respectively by

enhanced with three I shaped slots inscribe at the centre of 
Fig. 4 a Rectangular wearable patch antenna. b Antenna fixed with flexible clip

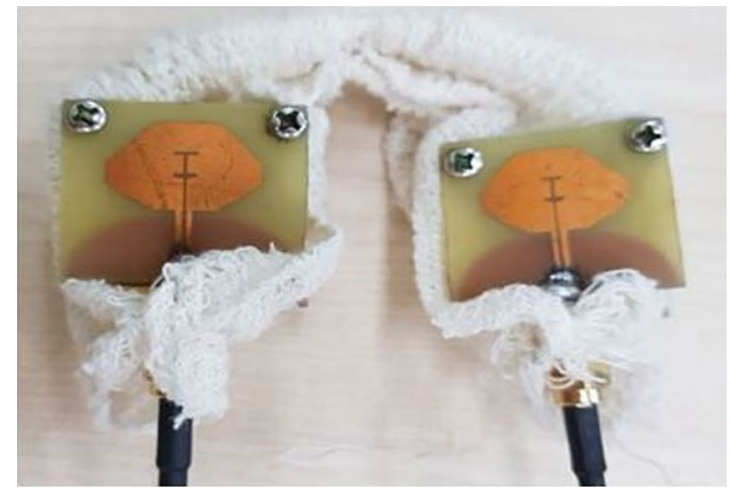

(a)

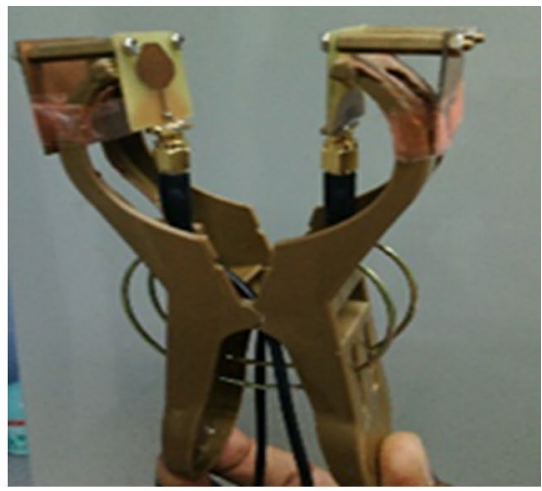

(b) maintaining the return loss $S_{11}<-10 \mathrm{~dB}$. These antennas were mounted over a clip and fixed to fit flexibly over ear lobe as shown in Fig. 4b.

PulsON ranging and communications module, P400 RCM [21] was used here as UWB transceiver. It is single board USB transceiver enables high precision distance measurement coupled with wireless data communication. Connection between antenna and transceiver were made with RG174 coaxial cable with a male/male SMA connector having impedance of $50 \Omega$ and length of $0.5 \mathrm{~m}$. Two Ethernet cables were used to connect PC with P400 RCM as can be seen in Fig. 3 .

\subsection{Software module}

Software module detail for the whole experiment is described as follows:

\subsubsection{Data acquisition}

Physical data were collected from students and local volunteers. All participants gave written consent prior to participate. For collection of actual blood glucose reading (BGCL) from participants (subject's), traditional Glucometer (ACCU-CHEK) was used.

The considered volunteers/human-subjects were within the age range from 20 to 65 years old, including both male $(\sim 50 \%)$ and female ( $50 \%)$. The day prior to data collection, the volunteers were briefed about data collection procedure, advised to have similar meals (content and amount) and fast overnight ( 8 to $10 \mathrm{~h}$ ). The next day, data collection was conducted in the morning (8-10 am) at university medical clinic, in a usual noisy room environment with temperature $24^{\circ} \mathrm{C}$ to $25^{\circ} \mathrm{C}$, in presence of doctors and nurses. After signing the consent form, the body temperature, blood pressure and pulse rate was measured for each volunteer. Then, an UWB signal was recorded through a volunteer's ear-lobe while the doctor drawn a blood sample from blood vessel from his/her hand. The collected blood samples were investigated clinically by doctors/medical staffs to measure actual $B G C L$ reading and compare with the output of the proposed system. The average thickness of earlobes of all human subjects was around $3.4 \mathrm{~mm}$ (Here to mention, the impact of the variation of earlobe thickness to $B G C L$ reading is under investigation, and not focus of this article. It is another thorough study and targeted for our next publication).

UWB transceiver generates and transmit the signal pulse with centre frequency of $4.7 \mathrm{GHz}$ from antenna placed one side of left ear lobe for each subject individually. Forward scattered pulses were recorded at the receiver antenna placed on the other side of the ear lobe as shown in Fig. 2. The transmitted UWB pulse sample and corresponding received samples depending on $B G C L$ is shown in Fig. 5. Collection of data physically (traditional) and through UWB system were done in parallel for each subject individually to make sure data consistency.

\subsubsection{Signal pre-processing and feature extraction}

Initially, the received analog signals were processed through segmentation. Then analog to digital conversion was done using discrete cosine transform (DCT) as shown in Eq. (1). Finally, each UWB pulse was converted to 1632 discrete data points. These heavy data were difficult to handle, hence feature extraction was considered for smooth and handy data processing towards achieving a light-weight and/or affordable system.

$F(n)=\left(\frac{2}{N}\right) \sum_{i=0}^{\frac{1}{2} N-1} \cos \left[\frac{\pi \cdot n}{2 \cdot N}(2 i+1)\right] \cdot X(i)$

where $N$ is the data samples and $X(i)$ is the received UWB signal. 


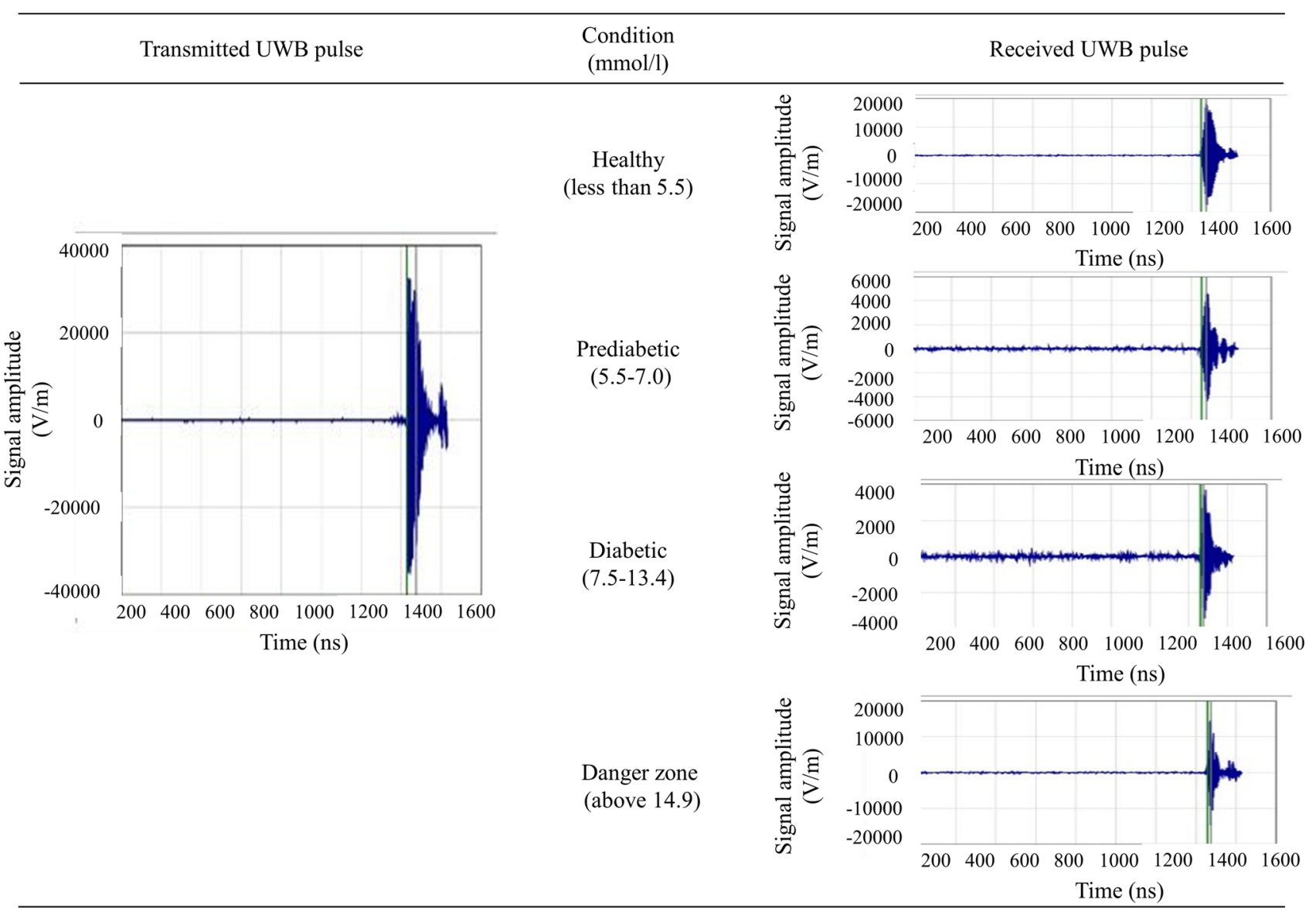

Fig. 5 One transmitted UWB pulse sample and corresponding received samples depending on BGCL status

\subsubsection{Feature extraction}

The negative effect of large number of data points to our system includes longer computational time, computational complexity, and handling difficulty. Saleh et al. [15], used principle feature analysis (PFA) extraction where 50-300 larger DCT values were considered instead of 1632. Reza [20] furthered enhanced Saleh's [15] method to reduce 1632 data points to four characteristic features, which are standard deviation (SD), mean $(\mu)$, maxima and minima using Eqs. (2) and (3) respectively. But their accuracy was not as expected due to the dependency of features mean and SD. To overcome that, we have considered all four independent features, which are SD, median, maximum and minima. These extracted four features are able to simplify the system with enhanced processing speed and reduced computational complexity.

$S D=\sqrt{\frac{1}{N-1}} \sum_{i=1}^{N}\left[A_{i}-\mu\right]^{2}$
$\mu=\frac{1}{N} \sum_{i=1}^{N} A_{i}$

$N$ represent total number of sample, $A_{i}$ is the number of data points and $\mu$ is the mean of $A, S D$ is the standard deviation.

\subsection{Deep artificial neural network model}

The four point independent features were then analysed for pattern recognition using MATLAB R2012a software. Feed forward back-propagation deep combination of artificial neural network (DANN) has been considered here, which is an enhancement of artificial neural network (ANN) [22] in terms of multiple layers and functions due to its simplicity and reasonable accuracy level. The hidden layers, and the number of neurons in hidden layers were tested from 20 to 4 to find the optimum value. The test was also conducted on other artificial intelligence (Al) models/algorithms [which are radial basis function (RBF) network, support vector machines (SVM), feed forward artificial neural 
network (ANN), random forest (RF), K-nearest neighbor (kNN), Naive Bayes (NB), and multilayer perceptron (MPL)] to find their efficiency, hence to justify our DANN model. The test results are presented in Table 1, which shows the efficiency in terms of converging time with lowest error (or highest accuracy) for each case. Therefore, the number of layers adjusted accordingly for all test cases.

Here, the employed algorithms to train the networks were Levenberg Marquart (trainlm), scaled conjugate gradient (trainscg), back-propagation (trainrp), deep combination (trainlm, trainscg and trainrp). Lastly, the mean square error Levenberg Marquart (trainlm) was used to enhance the entire training procedure.

Table 1 shows, our proposed DANN performances (BOLD), which is much better than other algorithms because we considered deep combination of hidden layers (multiple) and the training functions (trainrp, trainscg and trainlm), which contributes towards faster converging ( $0.2 \mathrm{sec}$, which is at least $50 \%$ less) with higher accuracy $(88.51 \%$, which is at least $10 \%$ increased) than other considered Al methods.

These best results were exhibited by $8 \times 8$ deep combination of DANN with eight (8) hidden layers and eight (8) neurons in each layer respectively. Figure 6 shows the developed DANN model with 8 hidden layer, four neurons as input (maxima, minima, median and SD) and one neuron in output layer. The used activation functions for input and output layers were tan-sigmoid and linear transfer function respectively. Equation (4) was used to get ANN regression.

$y=\sum_{i=1}^{n} w_{i} x_{i}+w_{0} x_{0}$

where $x, w$, and $y$ stands for input data set, weight vector and output value respectively. For respective algorithm, the value of $w$ was modified and adjusted according to the mean square errors (MSE) at the output level in each iteration. The MSE was calculated using Eq. (5).

$M S E=\frac{1}{N} \sum_{n=0}^{N-1}\left[y(n)-y_{m}(n)\right]^{2}$

where $y(n)$ and $y_{m}(n)$ represent the target and output respectively and $n$ is the number of used data points.

The total collected physical UWB data signals were 208, which was divided into three groups as follows:

(1) Training: 146 samples (70\%).

(2) Validation: 31 samples (15\%).

(3) Testing: 31 samples (15\%).
Table 1 Comparisons between artificial intelligence methods (algorithms)

\begin{tabular}{|c|c|c|c|c|}
\hline $\begin{array}{l}\text { Neural net- } \\
\text { work hidden } \\
\text { layers }\end{array}$ & Method/algorithm & $\begin{array}{l}\text { Converg- } \\
\text { ing time } \\
\text { (s) }\end{array}$ & Accuracy (\%) & Error (\%) \\
\hline 6 & Resource description framework (RBF) network & 0.76 & 78.35 & 21.65 \\
\hline 7 & Support vector machines (SVM) & 0.08 & 77.33 & 22.67 \\
\hline 7 & Artificial neural network (ANN) & 0.83 & 79.87 & 20.13 \\
\hline 8,8 & Deep combination of ANN (DANN) & 0.02 & 88.51 & 11.49 \\
\hline 9 & Random forest (RF) & 0.56 & 73.28 & 26.72 \\
\hline 10 & K-nearest neighbor (kNN) & 0.33 & 72.92 & 20.08 \\
\hline 11 & Naive Bayes (NB) & 0.04 & 75.20 & 24.80 \\
\hline 12 & Multilayer perceptron (MP) & 0.9 & 73.83 & 26.17 \\
\hline
\end{tabular}

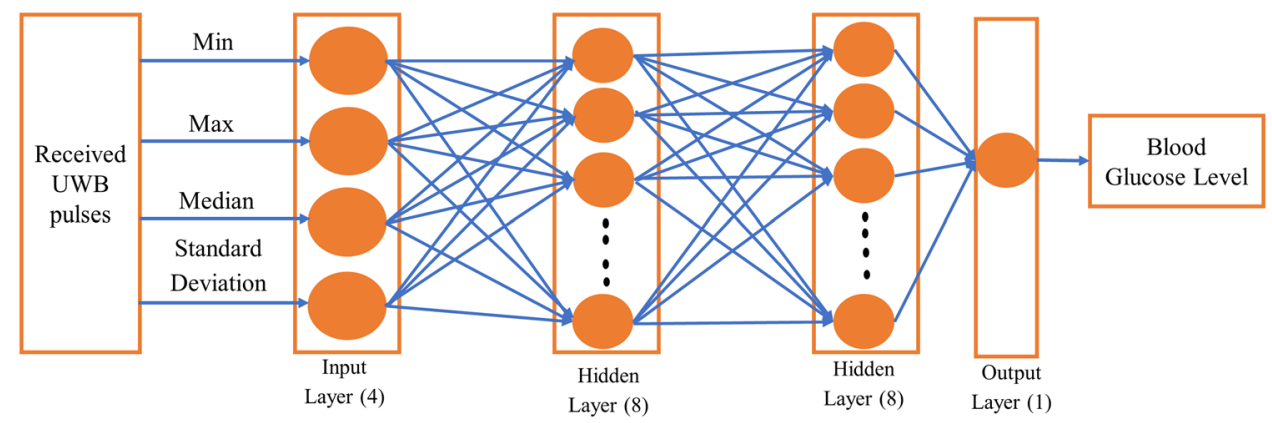


Table 2 Considered deep combination of ANN (DANN) parameters and functions

\begin{tabular}{ll}
\hline Neural network parameters & Value and functions \\
\hline Number of nodes in input layer & 4 \\
Number of hidden layers & 8 \\
Number of nodes in hidden layer & 8 \\
Number of nodes in output layer & 1 \\
Transfer function & tansig \\
Training function (deep combination) & trainlm, trainrp, trainscg \\
Maximum number of epochs & 1000 \\
Minimum performance gradient & $1 \mathrm{e}-25$ \\
Learning rate & 0.004 \\
Momentum constant & 0.9
\end{tabular}

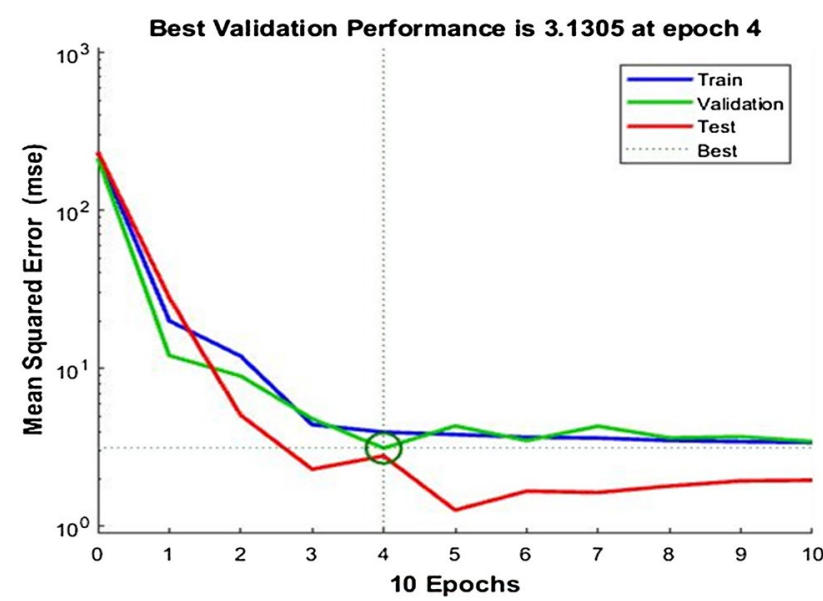

Fig. 7 Validation performance

\section{Results and discussions}

The DANN parameters and functions are shown in Table 2. Using these parameters and functions, the training, testing and validation performance of collected UWB data signals
Table 3 Relative accuracy of the system

\begin{tabular}{llll}
\hline $\begin{array}{l}\text { Investigation } \\
\text { type }\end{array}$ & $\begin{array}{l}\text { Average actual } \\
\text { value }\end{array}$ & $\begin{array}{l}\text { Average system } \\
\text { output }\end{array}$ & $\begin{array}{l}\text { Relative } \\
\text { accuracy }\end{array}$ \\
\hline BGCL $(\mathrm{mmol} / \mathrm{l})$ & 6.35 & 5.62 & 88.51 \\
\hline
\end{tabular}

is shown in Fig. 7 where best validation performance was observed at the 4th epoch with MSE value of 3.1305.

We wanted to observe the variation of DANN (system) output with the actual (physical/clinical) data. Accuracy of the system performance has been evaluated using Eq. (6). Here, average actual value means average of all $208 \mathrm{BGCL}$ samples collected physically using glucometer. The average system output indicates the average DANN output of 208 input signals. The average observed DANN output was $5.58 \mathrm{mmol} / \mathrm{I}$ which is $16 \%$ less than average actual measurement (average of 208 samples), as shown in Table 3.

Accuracy $=\frac{\text { system output }}{\text { actual value }} \times 100 \%$

Real time testing was done with 20 new datasets (not considered in training, testing and validation process) to confirm the accuracy level of the system. A portion of this testing (10 samples) is shown in Table 4.

The system shows consistent results with average accuracy of around $88 \%$, showing the system efficiency with reliability. Compared to other existing related technologies, this system is simple to operate and cost effective without any calibration prerequisite.

Human subjects have different body mussel composition with flash, skin, blood vessel, fat, bone etc. which cause deviation in change of dielectric properties. Received signals usually get affected by these artefacts. Previously this system was executed through hand mussel where the accuracy observed $82 \%$ [23]. Thus present result shows $6 \%$ improvement and confirms that use of DANN with the generation of signal through a body part with steady and sufficient blood contents exhibit a better performance. 
Table 4 Relative accuracy on real time blood glucose concentration level

\begin{tabular}{llllll}
\hline Data & $\begin{array}{l}\text { Actual reading } \\
(\mathrm{mmol} / \mathrm{l})\end{array}$ & $\begin{array}{l}\text { System output } \\
(\mathrm{mmol} / \mathrm{l})\end{array}$ & $\begin{array}{l}\text { Absolute } \\
\text { variation }\end{array}$ & $\begin{array}{l}\text { Relative accu- } \\
\text { racy (\%) }\end{array}$ & Average relative accuracy (\%) \\
\hline 1 & 5.0 & 4.713 & 0.29 & 94.26 & 87.62 \\
2 & 5.2 & 4.465 & -0.74 & 85.86 & (with 20 new data set) \\
3 & 7.3 & 8.38 & 1.08 & 85.21 & \\
4 & 5.4 & 6.25 & 0.85 & 84.32 & \\
5 & 8.5 & 7.11 & 1.39 & 83.62 & \\
6 & 10.2 & 8.65 & 1.55 & 84.78 & \\
7 & 12.5 & 10.71 & 1.79 & 85.68 & \\
8 & 6.7 & 7.62 & 0.92 & 86.21 & \\
9 & 4.5 & 4.558 & 0.66 & 98.71 & \\
10 & 7.0 & 7.87 & 0.87 & 87.51 & \\
\hline
\end{tabular}

\section{Conclusion}

The DANN and UWB based blood glucose level estimation and monitoring without using any blood sample is proposed here. This system works with approximately $88 \%$ accuracy, showing its suitability to be implemented in practice in near future. The system is non-invasive, user friendly in terms of GUI, affordable and is suitable to be used both by doctors at hospital and end users at home for regular BGCL measurement. In future, the numbers of pathological blood investigations can be computed through one system with having single input. Increased sample size, optimum deep learning Al and reduced signal to noise ratio while measuring data can have led to better accuracy, which is presently under investigation by our research team.

Acknowledgements This work is supported by Universiti Malaysia Pahang Internal Grant of RDU1703125. The authors would also like to thank Faculty of Electrical \& Electronics Engineering Technology, Universiti Malaysia Pahang (http://www.ump.edu.my) for providing the facilities to complete this work.

\section{Compliance with ethical standards}

Conflict of interest On behalf of all authors, the corresponding author states that there is no conflict of interest.

\section{References}

1. Statements P (2012) Standards of medical care in diabetes-2012. Diabetes Care 35(Suppl. 1):S11-S63

2. International Diabetes Federation (IDF) (2015) Diabetes Atlas. 7th Edition, International Diabetes Federation, Brussels, Belgium. Retrieved December 12,2019, from http://www.diabe tesatlas.org

3. Smith JL (2018) The Pursuit of Noninvasive Glucose: “Hunting the Deceitful Turkey" Sixth Edition. Retrieved December 12, 2019, fromhttps://www.researchgate.net/publication/32710
1583_The_Pursuit_of_Noninvasive_Glucose_Hunting_the Deceitful_Turkey_Sixth_Edition

4. Vashist SK (2012) Non-invasive glucose monitoring technology in diabetes management: a review. Anal Chim Acta 750:16-27

5. So CF, Choi KS, Wong TKS, Chung JWY (2012) Recent advances in noninvasive glucose monitoring. Med Devices Evid Res 5(1):45-52

6. Luaibi AY, Al-Ghusain AJ, Rahman A, Al-Sayah MH, Al-Nashash HA (2015) Noninvasive blood glucose level measurement using nuclear magneticresonance. In: 2015 IEEE 8th GCC Conference \& Exhibition, 1-4 Feb, Muscat, Oman, pp 1-4

7. Pulavskyi AA, Krivenko SS, Krivenko SA (2015) Noninvasive evaluation of glucose concentration in the human blood based on electrocardiograms. In: 2015 IEEE 35th international conference on electronics and nanotechnology, 21-24 April, Kiev, Ukraine, pp 275-277

8. Xiong X, Patra P, Hu J, Wu P (2016) Biochip with Graphene-based Nanosensor for Non-invasive Glucose Sensing Design. School of Engineering,University of Bridgeport, Bridgeport, CT 06604, pp 6604. Retrieved December 12, 2019, from https://core.ac.uk/ download/pdf/52956756.pdf

9. Malik S, Gupta S, Khadgawat R, Anand S (2015) A novel noninvasive blood glucose monitoring approach using saliva. In: 2015 IEEE International Conference on Signal Processing, Informatics, Communication and Energy Systems (SPICES), 19-21 Feb, Kozhikode, India, pp 1-5

10. Krivenko SS, Pulavskyi AA (2013) The new method for noninvasive measuring of glucose in human whole blood. Acad J Sci Res 1(4):69-81

11. Guo D, Zhang D, Zhang L, Lu G (2012) Non-invasive blood glucose monitoring for diabetics by means of breath signal analysis. Sens Actuators B Chem 173:106-113

12. Gerlitz J (2007) Non-invasive glucose meter. US7308293B2-Noninvasive glucose meter - Google Patents. Retrieved December 12, 2019, from https://patents.google.com/patent/US7308293B 2/en

13. Abdalsalam OS, Awouda AA (2014) Non-invasive glucose monitoring using scattering spectroscopy. Am J Biomed Eng 4(3):53-59

14. Naidis E, Mayzel $Y(2010)$ Noninvasive glucose monitoring: increasing accuracy by combination of multi-technology and multi-sensors. J Diabetes Sci Technol 4(3):583-595

15. AlShehri SA, Khatun S, Jantan AB, Raja Abdullah RSA, Mahmood $\mathrm{R}$, Awang Z (2011) Experimental breast tumor detection using $\mathrm{Nn}$-based Uwb imaging. Prog Electromagn Res 111:447-465 
16. Bilich CG (2010) Experiences on bio-medical sensing using ultra wideband communications and radar technology. Int J Ultra Wideband Commun Syst 1(4):248-262

17. Topsakal E, Karacolak T, Moreland EC (2011) Glucose-dependent dielectric properties of blood plasma. In: 2011 XXXth URSI General assembly andscientific symposium, 13-20 Aug, Istanbul, Turkey, pp 1-4

18. Karacolak T, Moreland EC, Topsakal E (2013) Cole-Cole model for glucose-dependent dielectric properties of blood plasma for continuous glucose monitoring. Microw Opt Technol Lett 55(5):1160-1164

19. Antonio P, Scarpelli PT, Rizzo M (1965) Small blood vessel involvement in diabetes mellitus: light microscope study of specimens obtained by ear lobe biopsy. Diabetologia 1(2):109-115

20. Reza KJ, Khatun S, Jamlos MF, Fakir M, Morshed MN (2015) Performance enhancement of ultra-wideband breast cancer imaging system: proficient feature extraction and biomedical antenna approach. J Med Imaging Health Inform 5(6):1246-1250
21. Time Domain Corporation, Comings Research Park, 330 Wynn Drive, Suite 300, Hantsville, Al 35805, USA

22. Azar AT (2013) Fast neural network learning algorithms for medical applications. Neural Comput Appl 23(3-4):1019-1034

23. Ali S, Khatun S, Kamarudin LM, Shoumy NJ, Vijayasarveswari V, Islam M (2016) Non-invasive ultra-wide band system for reliable blood glucose level detection. Int J Appl Eng Res 11(14):8373-8376

24. Ali MS, Shoumy NJ, Khatun S, Kamarudin LM, Vijayasarveswari V (2016) Non-invasive blood glucose measurement performance analysis throughUWB imaging. In: 2016 3rd International Conference on Electronic Design (ICED), 11-12 Aug, Phuket, Thailand, pp 513-516

Publisher's Note Springer Nature remains neutral with regard to jurisdictional claims in published maps and institutional affiliations. 